\title{
CONFIGURAÇÕES DO PROCESSO DE TRABALHO EM NÚCLEOS DE APOIO À SAÚDE DA FAMÍLIA E O CUIDADO INTEGRAL
}

\author{
WORK PROCESS CONFIGURATIONS IN FAMILY HEALTH \\ SUPPORT CENTERS AND COMPREHENSIVE CARE
}

\author{
CONFIGURACIONES DEL PROCESO DE TRABAJO EN NÚCLEOS DE \\ APOYO A LA SALUD DE LA FAMILIA Y EL CUIDADO INTEGRAL
}

\author{
Cynthia Maria Barboza do Nascimento ${ }^{1}$ \\ Paulette Cavalcanti de Albuquerque ${ }^{2}$ \\ Fabiana de Oliveira Silva Sousa ${ }^{3}$ \\ Luciana Camêlo de Albuquerque ${ }^{4}$ \\ Ide Gomes Dantas Gurgel
}

Resumo O estudo tem como objetivo sistematizar as configurações de Núcleos de Apoio à Saúde da Família. Trata-se de uma pesquisa qualitativa realizada em três municípios da Região Metropolitana do Recife, que utilizou dados coletados com grupos focais em três Núcleos de Apoio à Saúde da Família, analisados por meio da técnica de condensação de significados. Os resultados permitiram sistematizar três tipos de configurações: assistencial-curativista, que apresenta um distanciamento intenso do objetivo do Núcleo de Apoio à Saúde da Família; semimatricial, considerado uma configuração intermediária, e o matricial, que se aproxima mais do modelo ideal, preconizado pelo Ministério da Saúde. Conclui-se que existe, nos três municípios, uma proposta recente, frágil e que, apesar de ter potencialidades, é permeada por conflitos, contestações e incertezas. As informações obtidas podem subsidiar o planejamento e a execução de ações que busquem romper com vários conceitos hegemônicos norteadores da atuação dos profissionais de saúde na atenção básica.

Palavras-chave processo de trabalho; atenção básica; núcleo de apoio à saúde da família; equipe de saúde da família.
Abstract The aim of this study was to systematize the configurations of Family Health Support Centers. A qualitative study was conducted in three municipalities of metropolitan Recife, in the state of Pernambuco, Brazil, and considered data collected from focus groups in three Family Health Support Centers and analyzed through the meaning condensation technique. The study's findings allowed the systematizing of three types of configurations: the assistive-curative, which shows great distancing from the family health support center's goals; the semi-matrical, considered to be an intermediary configuration, and the matrical, which stays closer to the ideal model, recommended by the Ministry of Health. In conclusion, there is, on all three municipalities, a recent and fragile family health proposal that, despite its potentialities, is permeated with conflicts, contestations and uncertainties. The findings of the present study can assist in the planning and execution of actions that seek to break from various hegemonic concepts regarding the work of primary healthcare professionals.

Keywords work process; primary care; family health support center; family health team. 


\section{Introdução}

Com objetivo de apoiar a inserção da Estratégia Saúde da Família (ESF) na rede de serviços e de ampliar a abrangência, a resolubilidade, a territorialização e a regionalização das ações da Atenção Primária à Saúde no Brasil (APS), o Ministério da Saúde (MS) criou os Núcleos de Apoio à Saúde da Família (Nasf) (Brasil, 2008). Estes núcleos devem ser constituídos por equipes compostas por profissionais de diferentes áreas de conhecimento, para atuarem em parceria com a equipe de saúde da família.

O Nasf propõe repensar, transformar e apoiar a mudança da prática em saúde durante muitos anos vivida pela ESF. Portanto, em seus documentos normativos, o MS procurou embasar a proposta de Nasf e apresentou um arcabouço teórico, com diretrizes e dispositivos para construção e operacionalização do processo de trabalho.

Dentre estes dispositivos, foi apresentado o apoio matricial, que tem como principal objetivo garantir um apoio especializado a equipes e profissionais responsáveis pelo cuidado de problemas de saúde. Os apoiadores matriciais, que neste caso são os profissionais do Nasf, devem assegurar tanto retaguarda assistencial quanto técnico-pedagógica (Campos e Domitti, 2007).

O planejamento e o desenvolvimento do trabalho com base das dimensões assistencial e técnico-pedagógica buscam permitir que entre a equipe Nasf e a saúde da família aconteça um processo de trabalho dinâmico e interativo, no qual ocorra troca de experiências e conhecimentos, fato este que possivelmente contribuirá com a ampliação da clínica e potencializará a integração dialógica entre diferentes especialidades ou profissões de saúde (Cunha e Campos, 2011).

$\mathrm{O}$ dispositivo do apoio matricial vem paulatinamente ganhando visibilidade, à medida que passa a ser reconhecido como uma diretriz no Sistema Único de Saúde e incluído nas políticas de saúde criadas no Brasil. No entanto, apenas a inserção desta ferramenta nos serviços de saúde não garante a criação de sujeitos reflexivos, críticos e com uma maior autonomia no que se refere ao cuidado em saúde (Oliveira, 2008).

Muitos desafios surgem quando uma nova prática é introduzida a um processo de trabalho já organizado. A institucionalização de equipes com distintos profissionais integrando-se à equipe de saúde da família, que tem sua forma de atuação há certo tempo já organizada e consolidada, é considerada uma tarefa desafiadora, pois algumas rotinas têm que ser mudadas e com isso, desconfortos são gerados.

A perspectiva com a implantação do Nasf é de que, ao desenvolver um processo de trabalho claro, fixado nas diretrizes que tem sido propostas, e ao estimular ações interdisciplinares, a Atenção Básica consiga superar os seus 
desafios e ampliar suas fronteiras de atuação, com vistas a uma maior resolubilidade e integralidade da atenção.

Desde a criação do Nasf no Brasil, em 2008, existe uma inquietação e uma necessidade de se criar e consolidar espaços de discussões sobre como acontece o processo de trabalho dessa equipe com a da saúde da família, visto que a proposta aponta para uma diversidade de configuração e traz, ainda, questionamentos, incompreensões e, até mesmo, distorções.

A inovação da metodologia evidencia diferentes possibilidades de inserção e intervenção. Esse fato provoca reflexão com relação ao que é instituído e ao que é possível de ser construído e operacionalizado na rede de serviços loco regionais.

A complexidade da implementação dessa proposta exige que se concretize uma sistematização do conhecimento e uma aproximação dos diversos cenários, com o intuito de conhecer as realidades e enxergar as possibilidades encontradas para superar o desafio de ampliar a resolubilidade da ESF. Sendo assim, este trabalho tem como objetivo sistematizar os tipos de configurações de Núcleos de Apoio à Saúde da Família em três municípios da Região Metropolitana do Recife.

\section{Percurso metodológico}

Trata-se de uma pesquisa de abordagem qualitativa, com a realização de uma análise com base em múltiplos casos.

Foram usados, como cenário, três municípios do estado de Pernambuco descritos no texto como município $\mathrm{A}, \mathrm{B}$ e $\mathrm{C}$, intencionalmente selecionados em virtude de terem sido pioneiros na implantação do Nasf e possuírem uma rede de saúde capaz de estruturar e integrar as ações de atenção à saúde do usuário.

Os participantes do estudo foram profissionais de uma equipe de Nasf de cada município, a escolha se deu junto com coordenadores da atenção básica, e obedeceu aos seguintes critérios: funcionar por pelo menos dois anos; apresentar baixa rotatividade de profissionais e ter o processo de trabalho mais sistematizado.

Diante da necessidade de se obter diversas informações para embasar o estudo e potencializar as reflexões, o grupo focal foi escolhido por ser uma técnica que permite a coleta de dados em rodas de conversas coletivas entre diversos atores que apresentam características comuns de trabalho, de desejos e afetos (Westphal, Bógus e Faria, 1996).

A coleta de dados ocorreu no período de janeiro a maio de 2013. Foram formados três grupos focais, um de cada município. As conversas foram guiadas 
por um roteiro de temas e empregou-se uma metodologia com o objetivo de apresentar 'o que fazem' e 'como fazem', a fim de conhecer as ações e práticas desenvolvidas pelos profissionais do Nasf, com base nas discussões no coletivo.

Estes debates foram gravados, filmados e, ainda, registrados no diário de campo, tudo ocorreu com conhecimento e consentimento dos participantes, que assinaram o termo de consentimento livre e esclarecido. Para preservar a confidencialidade dos participantes, os grupos foram identificados da seguinte forma: Grupo Focal município A (GFA); Grupo Focal município B (GFB); Grupo Focal município C (GFC).

As falas produzidas nas sessões dos grupos focais de cada município foram transcritas, qualificadas e preparadas para análise do produto do texto, foi usado também o software Atlas-ti 5.0.

Para a análise do conteúdo dos grupos focais, foi adotada a técnica de condensação de significados do modelo de análise de entrevistas apresentados por Kvale (1996). A técnica consiste na sistematização dos significados encontrados no conteúdo, em especial, nas entrevistas. Tais significados são descritos de forma condensada, sem prejuízo de sua essência.

Por fim, para sistematização dos tipos de Nasf dos municípios estudados, foram considerados alguns aspectos, como: objetivo e formas de atuação, atividades realizadas, relação Nasf x ESF, articulação Nasf x outros serviços e ferramentas tecnológicas (Quadro 1).

Quadro 1

\begin{tabular}{|c|c|c|c|}
\hline $\begin{array}{l}\text { Aspectos } \\
\text { considerados }\end{array}$ & Nasf assistencial-curativista & Nasf equipe semimatricial & Nasf equipe matricial \\
\hline Objetivos do Nasf & Apoio de especialistas & Apoio de especialistas & $\begin{array}{l}\text { Suporte matricial às } \\
\text { equipes de saúde da família }\end{array}$ \\
\hline Forma de atuação & Assistencial & $\begin{array}{l}\text { - Assistencial } \\
\text { - Matriciamento com a equipe } \\
\text { de saúde da família }\end{array}$ & $\begin{array}{l}\text { - Matriciamento com a } \\
\text { equipe de saúde da família } \\
\text { - Integrada } \\
\text { - Interdisciplinar } \\
\text { - Promoção / prevenção } \\
\text { - Assistencial }\end{array}$ \\
\hline Atividades do Nasf & $\begin{array}{l}\text { - Atendimento individual na USF } \\
\text { - Atendimento individual na } \\
\text { policlínica } \\
\text { - Visita domiciliar } \\
\text { - Grupos de educação em saúde }\end{array}$ & $\begin{array}{l}\text { - Discussão de casos (Não } \\
\text { priorizada) } \\
\text { - Atendimento individual } \\
\text { - Atendimento compartilhado } \\
\text { - Visitas domiciliares } \\
\text { - Grupos de educação em } \\
\text { saúde }\end{array}$ & $\begin{array}{l}\text { - Discussão de casos } \\
\text { (priorizada) } \\
\text { - Atendimento individual } \\
\text { - Visitas domiciliares } \\
\text { - Grupos de educação em } \\
\text { saúde } \\
\text { - Educação permanente } \\
\text { - Preceptoria de alunos } \\
\text { - Reuniões entre serviços }\end{array}$ \\
\hline $\begin{array}{l}\text { Relação Nasf } x \\
\text { equipe de saúde da } \\
\text { família }\end{array}$ & Muito reduzida & Irregular & Regular e mensal \\
\hline
\end{tabular}




\begin{tabular}{|c|c|c|c|}
\hline \multicolumn{4}{|c|}{ Configuração dos tipos de Núcleos de Apoio à Saúde da Família } \\
\hline $\begin{array}{l}\text { Articulação Nasf x } \\
\text { outros serviços }\end{array}$ & Inexistente & Irregular & Regular e mensal \\
\hline $\begin{array}{l}\text { Ferramentas } \\
\text { tecnológicas }\end{array}$ & Não utiliza & $\begin{array}{l}\text { - Utiliza ocasionalmente o } \\
\text { apoio matricial }\end{array}$ & $\begin{array}{l}\text { - Apoio matricial } \\
\text { - Clínica ampliada } \\
\text { - PTS Projeto terapêutico } \\
\text { singular } \\
\text {-PST Projeto saúde } \\
\text { território } \\
\text { - Pactuação de apoio }\end{array}$ \\
\hline
\end{tabular}

Este artigo é resultado da tese de doutorado Núcleo de Apoio à Saúde da Família: uma análise da atenção à saúde em municípios da Região Metropolitana do Recife, apresentada ao Programa de Pós-Graduação em Saúde Pública do Centro de Pesquisas Aggeu Magalhães, Fundação Oswaldo Cruz. A pesquisa foi aprovada pelo Comitê de Ética do Centro de Pesquisas Aggeu Magalhães, mediante o certificado de apresentação para apreciação ética e obteve a aprovação sob o parecer n. 029/2012.

\section{Objetivo e formas de atuação do Nasf}

Na análise dos resultados foi possível verificar que os grupos têm posicionamentos diferentes quanto ao objetivo do Nasf. O GFC foi o que identificou seu trabalho como apoio matricial de fato e reconheceu a importância do trabalho em equipe, da troca de conhecimentos e experiências.

Nós não somos porta de entrada, trabalhamos como apoio. O momento mais rico do processo de trabalho do Nasf é a discussão entre as equipes, onde vamos repassando os conhecimentos específicos de cada um e, ao mesmo tempo, capacitando o Nasf e a equipe de saúde da família (GFC).

Os outros dois grupos GFA e GFB afirmaram que o principal objetivo era funcionar como apoio de especialistas aos usuários, ou seja, colocam o núcleo específico como principal responsável para resolver os problemas.

O usuário vai utilizar o Nasf como porta de entrada, então nós atuamos na base, na atenção básica e, lá, vamos distinguir ou direcionar este paciente para cada apoio específico (GFA).

(...) busca-se, com o Nasf, reduzir essa necessidade de um especialista, parece-me que isso é um dos objetivos do Nasf (GFB). 
Desta forma, percebe-se que a dificuldade de apropriação sobre o objetivo do Nasf pode ser um obstáculo para desenvolver um processo de trabalho baseado no matriciamento. O estudo de Andrade et al. (2012) também verificou que os participantes apontam o desconhecimento do papel do Nasf como uma das fragilidades da proposta. Para os autores, uma possível explicação é que as atividades da equipe de saúde da família e dos apoiadores matriciais ainda são desenvolvidas em conjunto com o modelo de Unidades Básicas de Saúde tradicionais e o Nasf é entendido como referência, e não como suporte. Nesse panorama, há uma sobrecarga de trabalho para os profissionais de saúde no desempenho das atividades previstas no modelo da ESF (como as visitas domiciliares, por exemplo), além daquelas características do modelo curativo-assistencial hegemônico.

Foi possível observar que o GFC referiu-se à atuação do trabalho em equipe e interdisciplinar, e que o Nasf deveria funcionar de forma integrada e articulada com a equipe de saúde da família.

Essa atuação é construída através da troca de conhecimento. Com ela, vários profissionais vão discutir as demandas vindas das unidades, esse é o diferencial, porque agora a unidade básica terá o suporte dos especialistas, isso vai facilitar e favorecer esse cuidado (GFC).

Com exceção do GFA, os grupos fizeram referência ao matriciamento como uma forma de atuação, consideraram que este dispositivo, se aplicado, pode ampliar o repertório de ações e potencializar a prática dos profissionais da saúde da família.

Você pode atuar a partir do matriciamento, matriciar as equipes em discussões de casos. É possível dar suporte, construir atividades com as equipes e formar grupos (GFC).

Nós atuamos, também, potencializando a equipe, fazendo consulta compartilhada com o profissional, é uma forma que nós temos de dar resposta para o caso - damos, assim, um potencial maior para a equipe (GFB).

Essa perspectiva de atuação baseada no matriciamento se aproxima do conceito e da prática apresentada por Campos e Domitti (2007). Os autores enfatizam que o objetivo da equipe de retaguarda é apoiar a equipe de referência na construção, reformulação e execução de cuidado à saúde. Refletem, ainda, que esse arranjo evidencia e assegura a importância na troca de saberes e a ampliação das ofertas de ações e serviços, na medida em que potencializa a ordem dos recursos disponíveis. 
Observou-se que foi comum para os grupos focais relacionar o Nasf à promoção da saúde e prevenção de doenças, embora relatassem que trabalhar nesta perspectiva é um grande desafio. Evidenciaram, ainda, a prática baseada no apoio assistencial.

Deveria existir o foco na promoção, na prevenção daquelas patologias. Mas, acredito que atuamos muito mais quando aquela demanda ou queixa chegam. Sobre a parte de promoção, acredito que ela seja pequena. Atuamos mais por encaminhamento e nessa parte curativa (GFB).

Nós realizamos as atividades em grupo, as discussões de casos se aproximam e trabalham mais a prevenção, que ainda é uma dificuldade na visão da unidade de saúde, pois ela sempre espera a atuação no curativo. Logo, trabalhar a promoção ainda é um desafio (GFC).

O Nasf é orientação. Entretanto, atuamos mais além, fazemos atendimentos, colocamos a mão na massa (GFA).

As diretrizes propostas pelo MS, mesmo de forma não suficiente, apresentam uma orientação normativa para que o Nasf não se configure como porta de entrada aos atendimentos individuais e ceda à pressão da imensa demanda reprimida. Mas, além disso, as diretrizes também precisam demarcar a necessidade de respostas a casos complexos que necessitem da intervenção direta de diferentes profissionais para a resolução do problema.

Nesse sentido, Cunha e Campos (2011) afirmam que a falta de serviços de saúde especializados pode conduzir a um funcionamento equivocado do Nasf, que assume um papel de substituição do serviço que falta, na tentativa de responder à demanda da população.

\section{Atividades dos Núcleos de Apoio à Saúde da Família}

A partir da análise dos grupos focais, verificaram-se quatro atividades principais desenvolvidas no cotidiano do Nasf: reuniões para discussões de casos, atividades em grupo, visitas domiciliares e atendimentos individuas - algumas dessas atividades foram destacadas como prioritárias. Vale salientar que, quando questionados sobre a definição de suas atividades, eles relataram outras ações, Além dessas quatro que apareceram de forma mais frequente.

A discussão de caso é priorizada e considerada um espaço importante, mas apenas pelo GFC, pois este grupo destacou que todas as outras atividades foram definidas nesse espaço da discussão; portanto, ela é uma atividade fixa na agenda das equipes. 
Construímos um cronograma mensal através das discussões de caso. É através da discussão que vão surgir as demandas de visita, os atendimentos e as demandas de grupo. A discussão de caso é uma atividade prioritária (GFC).

No entanto, eles enfatizam a dificuldade que se tem de garantir esses espaços e fazer com que eles aconteçam sistematicamente. No GFB e no GFA, as respostas foram muito vagas e as falas demonstraram que essa é uma atividade não priorizada e que não acontece sistematicamente.

É com a discussão de caso que nós nos reunimos, periodicamente, com as equipes de PSFs para discutir os casos e a própria relação do Nasf também. A periodicidade da discussão de caso... É bimensal? É Bimestral, geralmente. Tinha tudo para ser bimensal, mas geralmente é bimestral (GFB).

Então, estes espaços existem sim, mas eles estão sendo subutilizados, está lá garantido, mas não está acontecendo (GFA).

A falta de garantia de encontros sistemáticos referida pelos grupos acima, pode dificultar a relação entre o Nasf e a equipe de saúde da família. Campos e Domitti (2007) deixam claro que para existir de fato matriciamento é necessária a combinação de encontros periódicos e regulares.

Foi possível observar nas falas dos GFA e GFB que não existe a garantia de encontros sistemáticos de discussão entre as equipes, desta forma, os profissionais do Nasf tendem a organizar e desenvolver seu processo de trabalho de forma mais isolada, não se caracterizando como uma equipe de apoio à ESF. Fragelli (2013) também discutiu, em seu estudo, sobre a dificuldade dos profissionais se disponibilizarem para construir momentos de discussão em conjunto.

Para Campos (2012), é fundamental a existência de um espaço coletivo de formação de cogestão, em que sejam trabalhados: temáticas de interesse comum, discussão de casos clínicos, interpretações, análises, pactuações conjuntas, definição e planejamento de ações. Assim, é necessário que haja disponibilidade pessoal e institucional de todos os atores envolvidos, pois uma equipe que não está aberta para inserir em seu processo de trabalho momentos de encontro dificilmente conseguirá realizar o apoio matricial (Oliveira, 2011).

$\mathrm{O}$ atendimento clínico individual foi definido como uma atividade que faz parte do processo de trabalho de todos os grupos focais estudados, ou seja, os profissionais dos núcleos atendem às demandas específicas de cada categoria identificadas no território, pela equipe de saúde da família. No entanto, o GFA e o GFB evidenciaram que essa prática é a mais desenvolvida por eles quando comparada com outras. Foi destacado que não são 
eles quem priorizam os atendimentos específicos, essa demanda emerge das equipes de saúde da família e da cobrança por produção da própria gestão.

Eu não sinto que priorizo o atendimento individual, eu sinto uma grande demanda. As áreas em que nós atuamos são áreas que estão muito necessitadas do atendimento (GFB).

Eu tenho que completar 140 atendimentos ambulatoriais, 40 visitas e 12 grupos por mês (GFA).

Apesar de priorizarem o atendimento individual, a consulta compartilhada, que é realizada na Unidade de Saúde da Família ou na visita domiciliar com qualquer membro da ESF, também foi apontada como umas das atividades que podem ser desenvolvidas pelo Nasf.

Podemos atuar fazendo consulta compartilhada com o profissional. É uma forma que temos de dar resposta para o caso, damos um potencial maior para a equipe (GFB).

Todos os grupos focais assumiram a atividade de grupo como parte das ações cotidianas. No entanto, o GFC enfatiza que tanto a organização como a operacionalização do grupo são realizadas de forma compartilhada, entre profissionais da equipe Nasf e da equipe saúde da família, o que fortalece a dimensão do apoio matricial.

Nós vamos para as unidades onde fazemos grupos com os usuários. Fazemos os grupos e as ações, é muito gratificante pra a equipe e também pra os pacientes que procuram (GFA)

Priorizamos grupos. Até então nós, geralmente, somos demandados assim como diz nosso colega e acabamos por priorizar a participação em hiperdias, em grupos de idosos, de gestantes (GFB)

O grupo não é do Nasf. Primeiro, o grupo é organizado a partir da demanda da ESF, não somos nós que chegamos com a proposta, até porque não temos condição de fazer isso, não conhecemos a área, quem conhece a área e os seus os problemas é a equipe (GFC).

Alguns estudos apontam como tem sido o funcionamento do Nasf nos diversos municípios do Brasil. Silva et al. (2012), debateram a necessidade de uma definição inicial do processo de trabalho com base nas carências do território que se articulem às atividades das equipes de saúde da família. Os 
autores afirmam que foi consensual o fato de o formato não ser 'engessado', a fim de que, de acordo com a sua realidade e demanda, o Nasf possa ter autonomia para articular com as equipes de saúde da família e identificar as prioridades e a maneira de atuação.

O estudo propõe que a Secretaria Municipal de Saúde de São Paulo organize a atuação do Nasf, em três atividades, sendo elas: atendimentos específicos (5\% a 30\% da carga horária mensal), ações compartilhadas (35\% a $80 \%$ ) e ações intersetoriais ( $5 \%$ a $10 \%$ ). Além disso, os autores ressaltaram a potencialidade das reuniões para discussão de casos e o planejamento das ações (Silva et al., 2012).

A proposta de Bezerra et al. (2010) é de ampliar as ações em quatro categorias, e incorporar as reuniões para discussões de casos como uma atividade específica, são elas: reuniões mensais com as equipes de saúde da família; grupos educativos e terapêuticos; visitas domiciliares compartilhadas e atendimentos individuais e compartilhados.

Foram identificadas as mesmas ações citadas no parágrafo anterior e a necessidade de especificação de quais atividades respondem aos objetivos preconizados para o Nasf. No entanto, além de elencar, é importante equilibrar o desenvolvimento de práticas técnico-pedagógicas e assistenciais que atendam às demandas do território.

Um estudo realizado no Crato identificou que, apesar da proposta do Nasf estabelecer a orientação de trabalho em conjunto com a equipe de saúde da família, os profissionais não conseguiram se estruturar nesta lógica. Tendo em vista que, raramente, realizam atendimentos e visitas compartilhadas, as discussões de caso não são prioritárias, isto é, quando acontecem, ocupam os intervalos entre uma atividade e outra (Souza, 2013).

\section{Relação do Núcleo de Apoio à Saúde da Família versus Estratégia Saúde da Família}

Algumas equipes demonstraram que existem, de fato, uma aproximação maior e o desejo de um trabalho integrado; em outras, essa relação ainda é muito frágil ou, até mesmo, inexistente.

$\mathrm{O}$ não reconhecimento da equipe Nasf como um apoio foi um aspecto levantado pelo GFA como um fato que pode dificultar a interação entre as equipes. Verificou-se que a pouca disponibilidade para apoiar a saúde da família fragiliza a construção de vínculos e, consequentemente, não há a consolidação de uma relação entre as equipes.

A equipe de saúde da família não tem a aceitação do Nasf como grupo de apoio. Como costumamos dizer que 'pingamos' na unidade, a equipe diz: “O Nasf não 
vem aqui, então não faz parte da minha equipe." Teoricamente, não fazemos parte da equipe de saúde (GFA).

Observou-se que, no GFA, o Nasf não é reconhecido como uma equipe de apoio. A fala evidencia a fragilidade da relação entre as equipes, na qual o Nasf é identificado como fiscalizador, desenvolve ações fragmentadas, individualizadas, distanciadas, sem nenhum tipo de integração e corresponsabilização entre as equipes.

(...) O Nasf vem para analisar quem trabalha ou não e para cobrar. Se você chamar uma agente de saúde para fazer uma visita domiciliar e ela não for, eu preciso colocar no relatório. Então, essa é uma maneira de você fiscalizar a equipe de saúde. Com a equipe de saúde, eu não consigo ter essa adesão. Depende muito do 'chegar, fazer o seu e sair' (GFA).

Segundo o GFB, a relação com a saúde da família difere de uma equipe para outra. O grupo ressaltou que existe integração apenas com algumas equipes e, com outras, não é possível realizar o apoio, pois não existe um interesse por parte da equipe de saúde da família em atuar e praticar conjuntamente.

Em geral, vai bem. Há equipe mediana e existe a equipe que não funciona. É aquela coisa: você propõe algo e a equipe até topa, mas isso não quer dizer que ela vai fazer junto com você. Apesar disso, não atrapalha, faz ao modo dela (GFB).

A diversidade nas formas de atuação das equipes de saúde da família, algumas mais voltadas para ações de prevenção e promoção e outras mais para o atendimento individualizado, pode explicar as diferenças nas relações citadas acima. Para Aciole e Oliveira (2017) o grande desafio para construção de uma nova prática na atenção básica é romper com o modelo biomédico, e por isso a proposta de trabalho do núcleo tem provocado resistências em algumas equipes de saúde da família, que indicam, entre outros elementos, um distanciamento da realidade de trabalho e uma dificuldade no trabalho interdisciplinar exigido pelo apoio matricial, assim como pelo trabalho em saúde como um todo.

Ficou clara a vinculação e o reconhecimento da importância desta integração para o processo de trabalho no GFC. O grupo salientou que desenvolver um trabalho com base na garantia de uma relação efetiva entre equipes transforma a maneira como o profissional faz o cuidado à saúde.

Essa coisa de mudança paradigmática, que é uma mudança da maneira como você se relaciona, em como você se coloca. O problema não é mais da nutricionista, do 
psicólogo, o problema é das equipes, o sujeito é das equipes, por isso que a responsabilidade não é só dela, é nossa, isso é uma mudança de postura, acredito! (GFC).

Soares (2012) verificou, em seu estudo, que os processos de trabalho do Nasf fragmentam as ações e dificultam o estabelecimento de vínculo entre EqNasf e profissionais da saúde da família e os usuários. O estudo aponta, ainda, que as equipes de apoiadores matriciais substituir não são vistas como parte integrante da saúde da família. Desta forma, apresentam restrições para realizar atividades compartilhadas.

Neste estudo, verificou-se que as equipes enfatizam a necessidade de aproximação entre os profissionais do Nasf e da saúde da família, na tentativa de estreitar vínculos e fortalecer a atuação em conjunto. Fragelli (2013), em sua pesquisa, defende que trabalhar de modo colaborativo, mantendo interação e diálogo constante com os outros profissionais, foi um dos pontos levantados como competências e habilidades necessárias.

São muitos os desafios para a efetivação de práticas de integração entre os profissionais do Nasf, em contraste com aquilo que é esperado pela teoria que está na base dessa política de saúde. De fato, o trabalho só pode ser efetivado de forma integral se forem garantidas condições para o desenvolvimento da interprofissionalidade entre o Nasf e a saúde da família.

O desenvolvimento da prática interprofissional pode ser entendido como integração e articulação, na perspectiva da troca de experiências, com respeito aos saberes e à conduta de cada profissional, buscando estabelecer parcerias para transformar o cuidado em saúde (Batista, 2012).

No que refere à relação entre Nasf e equipe de saúde da família, Souza (2013) aponta que os profissionais do não podem ser enxergados como consultores, como especialistas que detêm o saber e estão naquele espaço para ensinar as equipes a lidarem com as dificuldades encontradas. Os profissionais do Nasf têm importante responsabilidade de ampliar a resolubilidade e a integralidade da saúde da família, esse fato pressupõe uma efetiva aproximação afetiva e relacional.

O diálogo surge como uma forma de reorganizar a relação entre os profissionais das equipes, o que facilita a pactuação e a corresponsabilidade. Se o diálogo não acontece, dificilmente haverá a possibilidade de ocorrer o apoio matricial.

Essa reorganização da forma de contato entre os profissionais é necessária para que a lógica do encaminhamento sem responsabilização seja superada (Figueiredo e Furlan, 2008). 


\section{Articulação do Núcleo de Apoio à Saúde da Família versus outros serviços}

Quando os grupos foram indagados sobre a relação entre os profissionais do Nasf e outros serviços, observou-se que todos entenderam a importância deste diálogo, porém apenas alguns conseguiram ter algum tipo de articulação e/ou comunicação.

O GFC mencionou que essa prática é uma atividade sistemática e garantida que faz parte do processo de trabalho da equipe. Já o GFB afirmou que é importante estreitar o vínculo com os serviços para fortalecer e qualificar as ações da saúde da família. Verificou-se, diante dos discursos, que essa articulação existe, mas ainda é incipiente, embora eles tenham relatado que estão em busca dessa troca.

As reuniões são frequentes, como núcleo de reabilitação nos encontramos uma vez por mês. (...) Há, ainda, as reuniões com os Caps [Centros de Atenção Psicossocial], está bastante estruturado, formalizamos isso numa reunião que tivemos com os Caps; enfim, está lá, existe um dia determinado e combinado com o serviço (GFC).

Podemos atuar intersetorialmente. Muitas vezes, nós temos um caso que vai além do Nasf, além da equipe de saúde. Então, precisamos, talvez, de uma intervenção do Caps, depende do caso, também fazemos essa ponte (GFB).

O GFA apontou ausência de relação sistemática com outros serviços; quando questionados, eles falaram sobre essa articulação por meio de capacitações e encontros realizados pela gestão.

A comunicação entre os serviços também surge como um dos aspectos favoráveis para potencializar as ações do Nasf. As diretrizes definidas pelo Ministério da Saúde apontam que a constituição de uma rede de cuidados é uma das estratégias essenciais dentro da lógica de trabalho da proposta, por isso a criação de espaços internos e externos de discussão entre os serviços da rede é tão importante. Todavia, quando não houver comunicação fácil entre a atenção terciária, secundária e primária, esta última não conseguirá exercitar seu papel na coordenação do cuidado (Mendes, 2010).

Prestes et al. (2011), em seu estudo realizado em Palmas, no estado do Tocantins, corroboram com as declarações dos grupos focais quando afirmam que a comunicação entre os serviços favorece a troca de conhecimentos, o compartilhamento das dificuldades e a corresponsabilização, e esses aspectos 
são essenciais para a oferta de serviços de saúde de qualidade. Constatou-se, ainda, que a garantia da articulação entre os serviços possibilita o resgate da relação entre os componentes da rede de saúde, estimula o diálogo cotidiano e contribui para um atendimento mais integral e resolutivo. Eles afirmam que a falta de comunicação entre os serviços compromete, significativamente, o funcionamento da rede de saúde.

Fragelli (2013) observou que apenas $26 \%$ dos participantes de seus estudos reconheceram que é uma habilidade da equipe de saúde da família e do Nasf articular os diversos níveis de atenção à saúde com a finalidade de contemplar as ações de promoção, de prevenção, de reabilitação e de cura. A autora diz que os profissionais enfatizaram que nem sempre há uma rede disponível para ser articulada.

\section{Ferramentas tecnológicas}

Quando as perguntas aos grupos foram sobre se conheciam e aplicavam as ferramentas tecnológicas foi consenso nos grupos a dificuldade em compreender esse termo. Fez-se necessário substituí-lo e exemplificar quais eram essas ferramentas: apoio matricial, clínica ampliada, projeto de saúde no território (PST), projeto terapêutico singular (PTS), e pactuação de apoio.

A clínica ampliada se caracteriza por estimular o desenvolvimento de ações baseadas em articulações de diversos enfoques e disciplinas. Ela estimula práticas baseadas no compartilhamento de saberes, experiências, condutas e procedimentos. Quanto ao PST, é considerado uma estratégia que deve ser desenvolvida de forma compartilhada entre a equipe Nasf e equipe de saúde da família, baseada em ações de produção da saúde em um território, com foco na articulação intersetorial, de forma a estimular a autonomia dos indivíduos e das comunidades (Brasil, 2009).

O PTS é considerado uma ferramenta que potencializa a prática interdisciplinar, pois se trata de um arranjo operador e gestor de cuidado, no qual acontece cooperação, trocas e compartilhamento de saberes centrado no paciente (Oliveira, 2007).

Nos discursos dos grupos focais, duas ferramentas emergiram de forma mais comum, foram elas: o matriciamento e o PTS; o projeto de saúde no território e a clínica ampliada foram citados rapidamente.

A clínica ampliada e o PTS também surgiram como ferramentas tecnológicas empregadas no processo de trabalho - a clínica foi apresentada como um mecanismo que perpassou todas as ações desenvolvidas. O GFC relatou, ainda, exemplos práticos da construção de PTS, ele enfatizou a importância dos projetos serem construídos em conjunto, considerando-se o conhecimento de cada ator que faz parte desse momento. 
A própria discussão já favorece a clínica ampliada e a troca de saberes.

Nós fazemos a construção do PTS nas discussões com a equipe. Toda a contribuição de todos os participantes é importante (GFC).

Com relação à clínica ampliada e ao PTS, as respostas foram muito confusas, porque as falas refletiram a dificuldade de entender a teoria e como essas ferramentas inseriram-se, de fato, no cotidiano dos profissionais da saúde.

O GFA, quando solicitado para falar sobre as ferramentas e sobre como elas foram utilizadas em suas ações, elencou apenas duas: o apoio matricial e a clínica ampliada. Percebeu-se, na fala, que o grupo relacionou a clínica ampliada a uma extensão de especialidades na atenção básica.

Primeiro, acredito que aquela clínica ampliada é uma ferramenta que fizemos, como havia falado, e que amplia o atendimento do médico, da enfermeira e dos outros colegas (GFA).

O MS define que o desenvolvimento das atividades do Nasf deve ser baseado no uso dos seguintes dispositivos: apoio matricial, clínica ampliada, projeto terapêutico-singular (PTS), projeto saúde no território (PST) e pactuação de apoio (Brasil, 2009). Esses dispositivos norteiam um modelo inovador e trazem aspectos diferentes dos conhecidos por muitos profissionais. Por esse motivo, geram estranhamentos e, consequentemente, dificuldades para serem incorporados na prática.

As discussões de casos, que seriam espaços para realizar o matriciamento e, assim, viabilizar o PTS e PST, na maioria das vezes, limitam-se à troca de guias de encaminhamento entre equipes. Muitas vezes, a reunião de apoio matricial acontece sem nenhum membro da equipe de saúde da família, o que torna um desafio inserir no processo de trabalho os dispositivos tecnológicos (Hori e Nascimento, 2014).

\section{Tipos de Nasf}

É importante deixar claro que essa tipificação não busca enquadrar o Nasf em certo ou errado, pelo contrário, o ideal é refletir quais estratégias são necessárias para uma atuação baseada no apoio matricial. Diante da análise dos eixos apresentados acima, definiram-se os seguintes tipos de Nasf concebidos em uma ideia de gradação, o que menos se aproxima da proposta, até o que funciona mais próximo do modelo preconizado pelo MS:

Nasf assistencial-curativista: atua como equipe de especialistas que atendem na atenção básica, apresenta um distanciamento intenso do objetivo do Nasf na medida em que usa o apoio apenas como um acesso aos especialistas. 
Nasf semimatricial: atua como equipe multiprofissional parcialmente integrada às suas equipes de referência; reflete a existência de configurações que apresentam ações assistenciais e técnico-pedagógicas, mas identifica-se uma priorização do atendimento ambulatorial em detrimento de outras ações conjuntas.

Nasf matricial: atua como equipe multiprofissional integrada às suas equipes de referência, aproxima-se mais do modelo ideal, ou seja, o preconizado pelo MS.

Os aspectos que configuram o 'Nasf assistencial-curativista' fazem referência a uma atuação de apoio de especialista focada no assistencial. Nele não foi possível reconhecer a atuação baseada nas diretrizes do Nasf. Existe, ainda, reduzida relação e pequena articulação das equipes dos Nasfs com as equipes de saúde da família e outros serviços da rede. A organização do processo de trabalho está voltada sempre para ações mais individuais (atendimento individual na USF, atendimento individual na policlínica, visita domiciliar), nas quais o profissional recorre apenas ao seu núcleo de saber para realizar intervenções e não faz trocas com outras disciplinas.

O estudo realizado por Araújo e Galimbertti (2013), em um município do Nordeste, também aponta para a atuação do Nasf focada no modelo curativo. Para os autores, a eficácia do Nasf está comprometida pelo efeito dispersivo provocado pelo arranjo organizacional que os sistema impõe. Com foco apenas no ambulatório, escolhe-se uma resposta reduzida, que não condiz com o que se espera da política de apoio à saúde da família.

Existem alguns desafios que precisam ser superados para minimizar a implementação do Nasf, segundo a configuração assistencial-curativista. Oliveira (2011), em seu estudo, aponta que o Nasf tem como objetivo realizar ações que superem o modelo biomédico, todavia ele esbarra na grande demanda reprimida que existe no território que necessita também de atenção individualizada de algumas especialidades. Soma-se a este fato a fragilidade da retaguarda assistencial das redes de atenção secundária.

Esta forma de atuação desvirtua o que é proposto pelo Nasf, principalmente no que se refere ao papel do apoio matricial, pois o que se observou nessa configuração é que não existe o apoio, e os profissionais limitam-se ao atendimento dos encaminhamentos feitos pela equipe de saúde da família e pelos gestores. Um estudo desenvolvido por Onocko-Campos et al. (2012) indicou que os principais desafios para a implantação do apoio matricial vão desde a dificuldade de entendimento da proposta até a falta de definição do perfil do necessário ao profissional responsável pelo apoio, além das falhas na organização dos encontros entre as equipes.

As deficiências na formação dos profissionais para as práticas na ESF extrapolam os saberes técnicos das profissões, uma das principais justificativas para o apoio matricial do Nasf. Capacitar os profissionais que hoje estão 
trabalhando na assistência é fundamental para que eles possam atuar de forma coerente com os princípios que norteiam a atenção básica (Ceccim, 2005; Ludke e Cutolo, 2010).

Bertussi (2010) afirma que os profissionais e a gestão consideram a implantação do Nasf como uma proposta de produção de serviços, ou seja, de especialistas que realizam ambulatório e não um espaço de articulação, apoio e produção de novas ofertas em parceria com as equipes de saúde da família. Este fato corrobora o que se observou no Nasf assistencial-curativista.

Não se verificou na organização do processo de trabalho desta configuração, uma atuação pautada no trabalho entre equipes. Ceccim e Ferla (2006) afirmam que o trabalho em equipe tem fundamental importância para a ampliação das compreensões e interpretações no cuidado.

Na configuração do 'Nasf semimatricial', observou-se fragilidade e confusão com relação às atividades realizadas, existe uma forma de apoio matricial, embora esta se realize ocasionalmente, ou seja, não está inserida no cotidiano das equipes e, ainda, não é vista como prioritária.

Foi possível verificar um estranhamento diante das novas práticas apresentadas pelo Ministério da Saúde e uma familiaridade com ações de caráter mais individual e menos integrado que, pelo fato de estarem na expectativa dos profissionais da saúde da família e do usuário, acabam priorizadas. Neste tipo, identificou-se uma aproximação incipiente, entre Nasf e equipe de saúde da família.

Existem aspectos fundamentais que precisam ser atribuídos ao Nasf para que ele seja considerado, de fato, uma equipe de apoio matricial. Na configuração definida na pesquisa como 'Nasf matricial', foi possível identificar características importantes que a aproximam do modelo proposto pelo MS

Aproximar-se do preconizado pelo MS não significa, evidentemente, que a mudança da proposta de trabalho buscada pelo apoio matricial esteja resolvida. O que se observou nos resultados desse estudo é que, de fato, houve um desencadeamento de um processo em que os encaminhamentos deixam de ser automáticos para poderem ser discutidos em equipes, mas isso, por si só, não garante que a ação seja compartilhada e integral.

O estudo desenvolvido por Bezerra et al. (2010) também apresenta resultados que demonstram uma aproximação do processo de trabalho com a proposta do MS. A pesquisa relata que o Nasf foi implantado com base na estratégia do apoio matricial. Entretanto, enfatiza que um dos maiores desafios é a superação da reprodução dos saberes e fazeres fragmentados, que limitam o papel do núcleo de cada profissional pela produção de novos padrões de inter-relação entre equipe e usuários e da quebra dos obstáculos organizacionais de comunicação.

É preciso discutir como construir esse apoio para que ele, efetivamente, potencialize transformações na prática dos profissionais, pois o modelo que 
sempre existiu no país, até a criação do SUS, teve seu foco na produção de atos de saúde como procedimentos sem comprometimento dos profissionais com a produção da promoção do cuidado (Merhy, 2003).

Esses tipos de Nasf identificados no presente estudo podem acompanhar a organização dos modelos da estratégia de saúde da família. Segundo Souza (2013), o engessamento das equipes de saúde da família pelos programas das áreas estratégicas básicas, o excesso de demanda, a fragilidade da rede de média e alta complexidade, a carência de recursos e a estrutura de trabalho são fatores que têm proporcionado a coexistência de dois modelos: um focado no assistencial, na doença e em procedimentos e um outro que busca alternativas para desenvolver a prática compartilhada e integral.

\section{Considerações finais}

Os resultados do estudo permitiram identificar que existe nos três municípios uma proposta recente, frágil e que, apesar de ter potencialidades, é permeada por conflitos, contestações e incertezas.

Em duas das tipificações apresentadas, falta alinhamento conceitual com relação aos dispositivos que orientam a atuação do Nasf, bem como há dificuldade de incorporá-los na produção do cuidado à saúde. A organização e o desenvolvimento do processo de trabalho diferenciam-se de um para outro; e até aquele município cuja configuração é do tipo matriciamento aponta dificuldades na sua operacionalização.

Considerando esses resultados e passados anos de implantação do Nasf no Brasil, a realização de avaliações sistemáticas no processo de trabalho é sugerida com o objetivo de conhecer sua resolubilidade, pois existem diferentes aspectos que influenciam na sua tipificação, ou seja, modificam-se conforme a região brasileira, mediante o território implantado e de acordo com as equipes que compõem a saúde da família e o Nasf, a política municipal, entre outros. Acredita-se que o processo avaliativo poderá ajudar a enfrentar os desafios e aprimorar a proposta.

Por fim, considera-se importante a realização do estudo, pois permite a ampliação do conhecimento sobre as dificuldades de implementação de um Nasf que contribua para a construção de um sistema de saúde mais resolutivo e integral.

Ademais, as informações obtidas podem subsidiar o planejamento e a execução de ações que busquem romper com vários conceitos hegemônicos norteadores da atuação dos profissionais de saúde e permitam atuar na atenção básica de forma interdisciplinar. 


\section{Colaboradores}

Cynthia Maria Barboza do Nascimento conduziu a pesquisa de campo e atuou em todas as etapas, desde a redação do projeto até a construção do artigo. Paulette Cavalcanti de Albuquerque e Ide Gomes Dantas Gurgel orientaram a tese de doutorado e auxiliaram na redação e na revisão crítica do artigo. Fabiana de Oliveira Silva Sousa e Luciana Camêlo de Albuquerque participaram da coleta de dados, análise, revisão e finalização do artigo. Não há conflito de interesses.

Resumen El estudio tiene como objetivo sistematizar las configuraciones de Núcleos de Apoyo a la Salud de la Familia. Se trata de una investigación cualitativa realizada en tres municipios de la Región Metropolitana de Recife, que utilizó datos recogidos con grupos focales en tres Núcleos de Apoyo a la Salud de la Familia, analizados por medio de la técnica de condensación de significados. Los resultados permitieron sistematizar tres tipos de configuraciones: asistencial-curativista, que presenta un gran distanciamiento del objetivo del Núcleo de Apoyo a la Salud de la Familia; semimatricial, considerada una configuración intermedia; y matricial, que se aproxima más al modelo ideal propuesto por el Ministerio de Salud. Se concluyó que en los tres municipios existe una propuesta reciente, frágil y que, a pesar de tener potencial, es atravesada por conflictos, discordancias e inseguridades. La información obtenida puede ayudar a la planificación y ejecución de acciones con el objetivo de romper varios conceptos hegemónicos que orientan la práctica de los profesionales de salud en la atención básica.

Palabras clave proceso de trabajo; atención básica; núcleo de apoyo a la salud de la familia; equipo de salud de la familia. 


\section{Notas}

${ }^{1}$ Universidade Federal de Pernambuco, Curso de Fonoaudiologia, Recife, Pernambuco, Brasil.

<cynthiabarboza@hotmail.com>

Correspondência: Avenida Santos Dumont, 1.500, apto. 3.204, Rosarinho, CEP 52050500, Recife, Pernambuco, Brasil.

${ }^{2}$ Fundação Oswaldo Cruz, Centro de Pesquisas Aggeu Magalhães, Recife, Pernambuco, Brasil.

<paulette.cavalcanti@gmail.com>

${ }^{3}$ Universidade Federal de Pernambuco, Recife, Pernambuco, Brasil.

<oliveirasilva.fabi@gmail.com>

${ }^{4}$ Prefeitura Municipal de Recife, Secretaria Municipal de Saúde, Recife, Pernambuco, Brasil.

$<$ luciana.camelo@gmail.com>

${ }^{5}$ Fundação Oswaldo Cruz, Centro de Pesquisas Aggeu Magalhães, Recife, Pernambuco, Brasil.

<ideg@cpqam.fiocruz.br>

\section{Referências}

ACIOLE, Geovani G.; OLIVEIRA, Dayana K. S. Percepções de usuários e profissionais da saúde da família sobre o Núcleo de Apoio à Saúde da Família. Saúde em Debate, Rio de Janeiro, v. 41, n. 115, out.-dez. 2017.

ANDRADE, Lucas M. B. et al.Análise da implantação dos Núcleos de Apoio à Saúde da Família no interior de Santa Catarina. Saúde e Transformação Social, Florianópolis, v. 3, n. 1, p. 18-31, jan. 2012. Disponível em: <http:// periodicos.incubadora.ufsc.br/index.php/ saudeetransformacao/ article/view/1471>. Acesso em: 22 mar. 2012.

ARAÚJO, Eliezer M. D.; GALIMBERTTI, Percy A. A colaboração interprofissional na Estratégia Saúde da Família. Psicologia \& Sociedade, Belo Horizonte, v. 25, n. 2, p. 461-468, maio 2013.
BATISTA, Nildo A. Educação Interprofissional em Saúde: concepções e práticas. Caderno FNEPAS, Rio de Janeiro, v. 2, jan. 2012.

BERTUSSI, Débora C. O apoio matricial rizomático e a produção de coletivos na gestão municipal em saúde. 2010. 234 f. Tese (Doutorado em Clínica Médica) -Faculdade de Medicina, Universidade Federal do Rio de Janeiro, Rio de Janeiro, 2010.

BEZERRA, Ricarda S. S. et al. Arranjo matricial e o desafio da interdisciplinaridade na atenção básica: a experiência do Nasf em Camaragibe/ PE. Divulgação em Saúde para Debate, Rio de Janeiro, n. 46, p. 51-59, 2010.

BRASIL. Ministério da Saúde. Secretaria de Atenção à Saúde. Departamento de Atenção Básica. Diretrizes do NASF: Núcleo de Apoio à 
Saúde da Família. Brasília, DF, 2009. (Cadernos de Atenção Básica).

BRASIL. Portaria GM n. 154, de 24 de janeiro de 2008. Cria Núcleos de Apoio a Saúde da Família-NASF. Diário Oficial [da] República Federativa do Brasil, Poder Executivo, Brasília, DF, 25 jan. 2008. Seção I, n.18, p. 47- 49.

CAMPOS, Gastão W. S. Apoiador matricial e práticas ampliadas e compartilhadas em redes de atenção. Psicologia em Revista, Belo Horizonte, v. 18, n. 1, p. 148-168, abr. 2012.

CAMPOS, Gastão W. S.; DOMITTI, Ana C. Apoio matricial e equipe de referência: uma metodologia para gestão do trabalho interdisciplinar em saúde. Cadernos de Saúde Pública, Rio de Janeiro, v. 23, n. 2, p. 399407, fev. 2007.

CECCIM, Ricardo B. Educação permanente em saúde: desafio ambicioso e necessário. Interface: Comunicação, Saúde, Educação, Botucatu, v. 9, n. 16, p. 161-168, set. 2004/fev. 2005.

CECCIM, Ricardo B.; FERLA, Alcindo A. Linha de cuidado: a imagem da mandala na gestão em rede de práticas cuidadoras para uma outra educação dos profissionais de saúde. In: PINHEIRO, Roseni; MATTOS, Ruben A. (orgs.). Gestão em redes: práticas, avaliação, formação e participação na saúde. Rio de Janeiro: Cepesc, 2006. p. 165-184.

CUNHA, Gustavo T.; CAMPOS, Gastão W. S. Apoio matricial e atenção primária em saúde. Saúde e Sociedade, São Paulo, v. 20, n. 4, p. 961-970, 2011.

FIGUEIREDO, Mariana D.; FURLAN, Paula G. O subjetivo e o sociocultural na co-produção de saúde e autonomia. In: CAMPOS, Gastão W. S.; GUERRERO, André V. P. Manual de práticas de atenção básica: saúde ampliada e compartilhada. São Paulo: Hucitec, 2008. p. 154-178.
FRAGELLI, Thaís B. O. Análise das competências profissionais no núcleo de apoio à saúde da família. 2013. 176f. Tese (Doutorado) - Faculdade de Ciências da Saúde, Universidade de Brasília, Brasília, DF, 2013.

HORI, Alice A.; NASCIMENTO, Andréia F. Projeto Terapêutico Singular e as práticas de saúde mental nos Núcleos de Apoio à Saúde da Família (Nasf) em Guarulhos/SP. Ciência \& Saúde Coletiva, Rio de Janeiro, v. 19, n. 8, p. 3561-3571, ago.2014. Disponível em: $<$ http://www.cienciaesaudecoletiva.com.br/ artigos/artigo_int.php?id_artigo $=14501>$. Acesso em: 10 jan. 2015.

KVALE, Steinar. Inteviews: an introduction to qualitative research interviewing. London: Sage, 1996.

LUDKE, Luise; CUTOLO, Luiz R. A. Formação de docentes para o SUS: um desafio sanitário e pedagógico. Saúde \& transformação social, Florianópolis, v. 1, n. 1, p. 88-98, 2010.

MENDES, Eugênio V. As redes de atenção à saúde. Ciência \& Saúde Coletiva, Rio de Janeiro, v. 15, n. 5, p. 2297-2305, 2010.

MERHY, Emerson E. et al. O trabalho em saúde: olhando e experienciando o SUS no cotidiano. 2. ed. São Paulo: Hucitec, 2003.

OLIVEIRA, Gustavo N. O projeto terapêutico como contribuição para a mudança das práticas de saúde. 2007. Dissertação (Mestrado em Saúde Coletiva - Planejamento, Gestão e Subjetividade) - Faculdade de Ciências Médicas, Universidade Estadual de Campinas, Campinas, 2007.

OLIVEIRA, Gustavo N. Apoio matricial como tecnologia de gestão e articulação em rede. In: CAMPOS, Gastão W. S.; GUERRERO, André V. P. Manual de práticas de atenção básica saúde ampliada e compartilhada. São Paulo: Hucitec, 2008. p. 273-281. 
OLIVEIRA, Gustavo N. Devir apoiador: uma cartografia da função apoio. 2011. 168f. Tese (Doutorado) - Universidade Estadual de Campinas, Faculdade de Ciências Médicas, Unicamp, 2011.

ONOCKO-CAMPOS, Rosana T. et al. Avaliação de estratégias inovadoras na organização da Atenção Primária à Saúde. Revista de Saúde Pública, São Paulo, v. 46, n. 1, p. 43-50, 2012.

PRESTES, Ludimila I. N. et al. Apoio matricial: um caminho de fortalecimento das redes de atenção à saúde em Palmas-TO. Revista Brasileira de Ciências da Saúde, João Pessoa, v. 15, n. 2, p. 215-218, 2011.

SILVA, Andréa T. C. et al. Núcleos de Apoio à Saúde da Família: desafios e potencialidades na visão dos profissionais da Atenção Primária do Município de São Paulo, Brasil. Cadernos de Saúde Pública, Rio de Janeiro, v. 28, n. 11, p. 2.076-2.084, 2012.
SOARES, Gisele. M. M. Trabalho em equipe multidisciplinar: concepções e práticas dos profissionais dos Núcleos de Apoio à Saúde da Família. 2012. Monografia (Especialização em Saúde Pública) - Universidade Estadual do Ceará, Fortaleza, 2012.

SOUZA, Fernanda R. Processo de construção dos Núcleos de Apoio à Saúde da Família (NASF) na atenção básica do estado do Ceará. 2013. 220f. Dissertação (Mestrado em Saúde Pública) - Universidade Federal do Ceará, Faculdade de Medicina, Fortaleza, 2013.

WESTPHAL, Márcia F.; BÓGUS, Cláudia M.; FARIA, Mara M. Grupos focais: experiências precursoras em programas educativos em saúde no Brasil. Boletín de la Oficina Sanitaria Panamericana, Washington, v. 120, n. 6, p. 472-482, 1996.

Recebido em 21/08/2017.

Aprovado em 07/02/2018 